\title{
Unified Particle Model for Multiple-fluid Flow and Porous Material
}
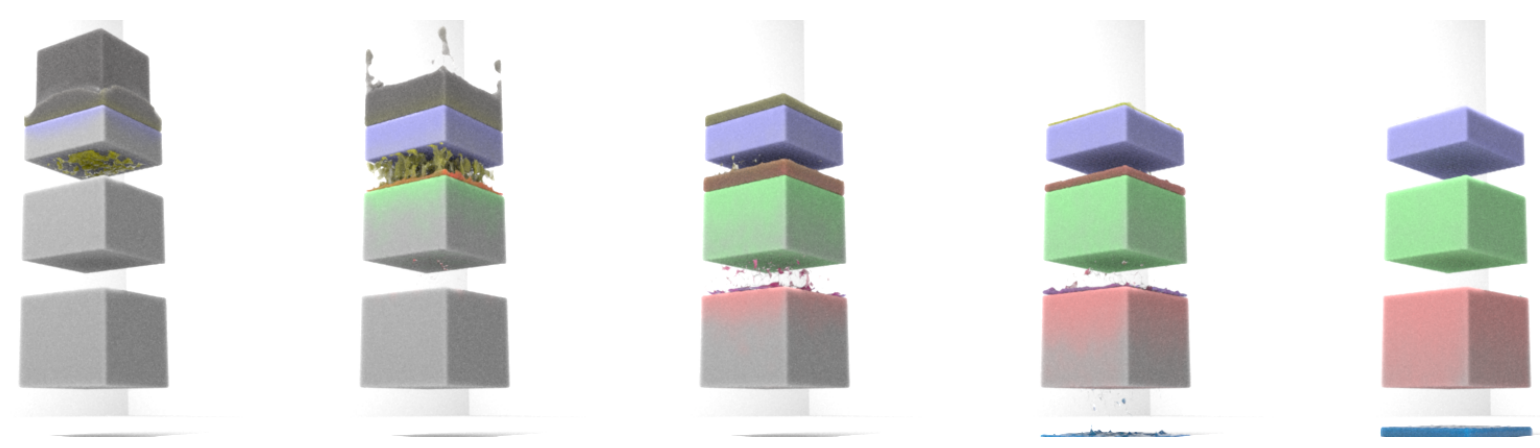

Figure 1: Filtering of three-phase liquid mixture. The liquid falls through three solid layers in a tunnel, with each layer absorbing one of the phases but letting other two phases pass through. The three phases are separated from originally mixed state and each block shows the corresponding absorbed phase colour.

\begin{abstract}
Porous materials are common in daily life. They include granular material (e.g. sand) that behaves like liquid flow when mixed with fluid and foam material (e.g. sponge) that deforms like solid when interacting with liquid. The underlying physics is further complicated when multiple fluids interact with porous materials, involving Darcy's law, Navier-Stokes equations, and poroelasticity etc. We propose a unified particle model for the simulation of multiple-fluid flows and porous materials. A novel virtual phase concept is introduced to avoid explicit particle state tracking and runtime particle deletion/insertion. Our unified model is flexible and stable to cope with multiple fluid interacting with porous materials, and it can ensure consistent mass and momentum transport over the whole simulation space.
\end{abstract}

\section{CCS CONCEPTS}

- Computing methodologies $\rightarrow$ Physical simulation.

\section{KEYWORDS}

Porous material, smoothed particle hydrodynamics, multiple fluids, virtual phase

\section{INTRODUCTION}

Unlike impermeable materials that can only interact with fluid flows via their surfaces, porous materials present a much stronger coupling when exposed to fluids, and the solid-fluid interaction takes place both on their surfaces and inside their bodies. Phenomena such as water spilling out of a squeezed sponge, sea wave splashing on a sandy beach and cotton toy deforming when wetted are common examples in daily life. These flow phenomena are far more complicated than the single-phase fluid flow in an open space, and they could involve many physical processes occurring simultaneously, such as liquid mass transport in the porous solid, absorption

Author's address: and emission on the solid surface, hydrophobic and hydrophilic behaviours, capillary effects, and multiphase flows etc.

Simulations of single-fluid flow involving porous materials have been achieved using SPH (smoothed particle hydrodynamics) [Lenaerts et al. 2008] and MPM (material point method) [Tampubolon et al. 2017], producing impressive visual results. Despite the success, both methods face challenges associated with porous behaviours. Using the SPH framework [Lenaerts et al. 2008], the absorption and emission of liquid is modelled by deleting/inserting fluid particles and tracking the fluid flow inside the solid particles; thus, the simulator must constantly delete/insert non-uniform SPH particles, a process that is unstable and prone to error. A naive extension of this approach to multiple fluid simulation will involve splitting the fluid particles as different fluid phase can potentially interact with the solid in different ways. This will be an exhausting process and also result in non-uniform particle sizes. Alternatively, without splitting the fluid particle, one needs to cope with different mass and momentum transports among various phases and maintain consistency for fluid particles crossing the solid boundary. The MPM approach [Tampubolon et al. 2017] uses two layers of grid to model sand and liquid motions separately. For the sand-like porous material, a similar set of Navier-Stokes equations applies both to the pure liquid phase and the solid-liquid mixture so that the focus is on the interaction between these two grid layers. However for other porous materials like sponge, the fluid motions inside and outside the porous solid can differ greatly, and other physical processes like capillary effects may also be involved, so that to extend the capacity of the multi-grid MPM approach is not a trivial task.

In this paper we present a universal SPH-based simulation scheme for multiple-fluid flows in porous materials. A special emphasis is on sponge-like materials where the internal flow follows Darcy's law and external flow is governed by the Navier-Stokes equations. In our approach, the internal and external flows are modelled uniformly using the volume fraction and a mixture model, avoiding constant particle deletion and insertion. The new approach is able 
to capture a wide range of porous media flow phenomena, including poroelasticity, capillary effects and variable absorption in multiplefluid environment. Our main contributions are:

- A universal SPH framework for the simulation of multiplefluid flow and sponge-like porous materials.

- A novel concept of virtual phase to consistently capture the fluid flow when crossing porous surface and avoid runtime particle deletion/insertion.

- A flexible constitutive modelling strategy based on the virtual phase concept, to support multiphase flow, porous material deformation and the coupled physics inside, outside and on the porous surface.

The rest of paper is arranged as follows. Related works are briefly recapped in $\S 2$, after which the fundamental theories of our multiple-fluid SPH-based porous model is introduced in $\S 3$. We explain the constitutive models in $\S 4$, and implementation issues in $\S 5$. A number of examples with different visual effects are presented in $\S 6$ to demonstrate the performance of the new model. Finally, concluding remarks and limitations are discussed in $\S 7$.

\section{RELATED WORK}

There are a variety of porous material theories. Some physics research works explicitly model the detailed geometry of porous materials [Berrone et al. 2017; Hilfer 2006; Wu et al. 2004]. They treat a porous material as a network of connected pore spaces and track the fluid flow in the pore-space network by carefully calculating the interactions between pore surface and fluid. The graphics community, on the other hand, usually uses macroscopic representations and avoids directly modelling complex microscopic fractures. As a result, the fluid motion generally obeys different laws inside and outside the porous solid. [Lenaerts et al. 2008] used an SPH-based method to couple the fluid/solid motion and the absorption/emission by porous material. Darcy flux was introduced to model the absorbed flow behaviour inside the porous material. This model can be applied to a variety of scenarios involving porous media flow, but it requires frequent particle deletion and insertion, which is inaccurate and unstable in practice.

As a special case of porous media flow, the granular flow has been receiving more attention from the graphics community in recent years. [Yan et al. 2016; Yang et al. 2017] used the volume fraction to describe the concentration of granular material in the liquid, and the porous behaviour was treated similarly to solid dissolving. [Gao et al. 2018; Tampubolon et al. 2017] used MPM to simulate granular sand flows in water, and two background grids were used for the fluid and sand phase separately, with the interaction between phases handled through a momentum exchange term. This approach can handle large scenarios effectively, but does not cover wider physics, e.g. capillary phenomena. In addition, the previous research works of granular materials usually use Navier-Stokes equations for both the pure liquid phase and the solid-liquid mixture, while the internal flow could be better captured by using Darcy's law.

\section{UNIVERSAL SPH MODEL FOR MULTIPHASE FLOW AND POROUS MATERIAL}

A simulation space involving porous materials and fluid flows naturally divides into two parts, i.e. the "inner" and "outer" regions of the porous material, and any fluid flow in the simulation space can be considered as travelling in or between these two regions. Previous approaches typically use two types of SPH particles to represent the porous solid and the fluid respectively, with the fluid particles placed only in the outer region. As a result, SPH particles are deleted or inserted when fluid passes through the surface of porous material. To avoid the loss of accuracy and stability, we propose a universal SPH scheme where fluid particles remain in the simulation when they cross the surface of porous material (whether through absorbing or emitting) and unlike previous methods, no particle gets deleted or inserted as a result of crossing interface. This is achieved by a novel extension of the volume fraction representation and the mixture model [Ren et al. 2014], and the details are explained below.

\subsection{Governing Equations}

We propose to use three sets of equations to capture the coupled physics of multiple-fluid flow and porous materials. First, for fluid flow inside the porous material, we use Darcy's law [Darcy 1856] to simulate the flow motion. Specifically, the instantaneous flow rate, i.e. Darcy flux, in a homogeneous permeable material is determined by

$$
\boldsymbol{q}_{k}=-\frac{\boldsymbol{k}_{\boldsymbol{w} \boldsymbol{k}}}{\mu_{k}} \cdot \nabla p^{\text {pore }}
$$

where the subscript $k$ denotes the $k$-th fluid phase, $\boldsymbol{q}_{k}$ Darcy flux of the phase $k, \boldsymbol{k}_{\boldsymbol{w} \boldsymbol{k}}$ the permeability tensor, $\mu_{k}$ the fluid viscosity, and the pore pressure $p^{\text {pore }}$ represents the pressure of fluid in the pore space.

Next, for fluid flow outside the porous material, we adopt the multiple-fluid mixture model [Ren et al. 2014; Yan et al. 2016]:

$$
\begin{gathered}
\frac{D \tilde{\alpha}_{k}}{D t}=-\tilde{\alpha}_{k} \nabla \cdot \boldsymbol{u}_{m}-\nabla \cdot\left(\tilde{\alpha}_{k} \tilde{\boldsymbol{u}}_{m k}\right) \\
\frac{\partial}{\partial t} \boldsymbol{u}_{m}+\left(\boldsymbol{u}_{m} \cdot \nabla\right) \boldsymbol{u}_{m}=\boldsymbol{g}+\boldsymbol{a}^{\text {press }}+\boldsymbol{a}^{\text {other }}
\end{gathered}
$$

where $\tilde{\alpha}_{k}$ is the volume fraction of phase $k, \boldsymbol{u}_{m}$ and $\tilde{\boldsymbol{u}}_{m k}$ are the mixture velocity and drift velocity for the phase $k$, and $\boldsymbol{g}, \boldsymbol{a}^{\text {press }}$ and $\boldsymbol{a}^{\text {other }}$ denote the gravity, pressure, and other forces (viscosity, etc.), respectively.

Finally, for the porous solid, we simply adopt the elastic solid model [Peer et al. 2018] and the strain tensor $\boldsymbol{\epsilon}$ is expressed as:

$$
\boldsymbol{\epsilon}=\frac{1}{2}\left(\boldsymbol{F}+\boldsymbol{F}^{T}\right)-\boldsymbol{I}
$$

where $\boldsymbol{F}$ is the deformation gradient tensor and $\boldsymbol{I}$ is the identity matrix. To take into account the effect of pore pressure on the deformation of solid, we express the stress tensor $\boldsymbol{P}$ as follows:

$$
\boldsymbol{P}=2 \mu \boldsymbol{\epsilon}+\lambda \operatorname{tr}(\boldsymbol{\epsilon}) \boldsymbol{I}-\eta p^{\text {pore }} \boldsymbol{I}
$$

where $\mu, \lambda$ and $\eta$ are material constants, and the last term represents the effect of pore pressure. 


\subsection{Virtual Phase for Fluid Particles}

The above governing equations can be solved individually with existing methods, but they become strongly coupled near the surface of porous material, greatly increasing the solution complexity. We propose the concept of virtual phase to handle the state change of fluid particles crossing the interface and the associated mass and momentum transport in a uniform manner, thereby avoiding explicit state tracking and runtime particle deletion/insertion.

Specifically, we assign each fluid particle with two virtual phases $\alpha_{k}$ and $\beta_{k}$, which represent the volume fractions of the phase $k$ in the outer and inner regions, respectively, and they satisfy the relation $\sum_{k}\left(\alpha_{k}+\beta_{k}\right)=1$. Thus, $\sum_{k} \alpha_{k}=1$ indicates the fluid particle located outside the porous material, $\sum_{k} \beta_{k}=1$ indicates the particle located inside the porous material, and non-zero $\alpha$ and $\beta$ values indicate the fluid particle is near the surface of porous material. Consider an example where a fluid particle at a two-phase state is moving from the outer region into the inner region, but only one fluid phase can be absorbed into the porous material while the other cannot. The mass transport, momentum and position change of this fluid particle are affected by all material phases, each in turn may have separate fractions following different laws in the inner and outer regions. The virtual phase concept distinguishes these fractions and virtual phases can follow separate governing equations. The virtual phases have a property that $\alpha_{k}$ and $\beta_{k}$ can be transferred, by absorption or other mechanisms, near the surface of porous material without violating the mass conservation law, but volume fractions between real phases cannot be simply transferred.

As a result of porous material and fluid interaction, the fluid particle motion and its (virtual) phase composition change constantly as it moves from one zone to another, and it can be further complicated when multiple fluids are involved and each behaves differently in relation to the porous solid. To capture such complex coupling, we extend the mixture model using the virtual phase concept:

$$
\begin{gathered}
\frac{D \alpha_{k}}{D t}=-\alpha_{k} \nabla \cdot \boldsymbol{u}_{m}-\nabla \cdot\left(\alpha_{k} \boldsymbol{u}_{m k}\right)+\nabla \cdot \boldsymbol{D}_{\boldsymbol{k}} \\
\frac{D \beta_{k}}{D t}=-\nabla \cdot\left(\beta_{k} \boldsymbol{u}_{\beta k}\right)+\boldsymbol{u}_{m} \cdot \nabla \beta_{k}-\nabla \cdot \boldsymbol{D}_{\boldsymbol{k}} \\
\frac{\partial}{\partial t} \boldsymbol{u}_{m}+\left(\boldsymbol{u}_{m} \cdot \nabla\right) \boldsymbol{u}_{m}=\boldsymbol{g}+\boldsymbol{a}^{\text {press }}+\boldsymbol{a}^{\text {pore }}+\boldsymbol{a}^{\text {cap }}+\boldsymbol{a}^{\text {visc }}
\end{gathered}
$$

where $\alpha_{k}$ and $\beta_{k}$ are the outer and inner volume fractions of phase $k, \boldsymbol{u}_{m}$ and $\boldsymbol{u}_{m k}$ are the fluid-particle velocity and drift velocity, $\nabla \cdot D_{k}$ is a absorption source term, $\boldsymbol{v}_{\beta k}$ is the pore velocity of the inner part of phase $k$, and $\boldsymbol{g}, \boldsymbol{a}^{\text {press }}, \boldsymbol{a}^{\text {pore }}, \boldsymbol{a}^{\text {cap }}, \boldsymbol{a}^{\text {visc }}$ denote the gravity, pressure, pore pressure, capillary force and viscosity, respectively. The extended mixture model in Eqns. $(6,7,8)$ can be applied to all fluid particles and ensure physically consistent mass and momentum transport when fluid particles enter and exit the solid region. A brief explanation is given below followed by more details in $\S$ ??.

Eqn. (6) and Eqn. (7) are the continuity equations, where the outer and inner fluids are separately calculated since they follow different mass transportation laws. For the inner fluid $\beta_{k}$, Darcy's law directly gives the phase-wise porous velocities, so that Eqn. (7) does not use the drift velocity but the phase velocity instead. The drift velocity label $\boldsymbol{u}_{m k}$ is now used only for outer-region particles.
In Eqn. (8), a pure outer particle is not affected by the pore pressure calculated in the inner region and the capillary effect near the solid surface, so these terms are zero; a pure inner particle has no pressure and viscosity forces; and for a hybrid particle, both inner and outer force sources are calculated and included in the particle motion computation.

\section{CONSTITUTIVE MODELS}

Besides the momentum equations described in $\S 3.1$, the behaviours of multiphase flow and porous media are mainly determined by their constitutive models. Based on the virtual phase concept, we formulate a set of coherent SPH models to capture the different physical processes occurring in the inner region, in the outer region, and on the porous surface, which are explained in $\S 4.1, \S 4.2$ and $\S 4.3$, respectively.

\subsection{Inner Region Physics}

The momentum balance in the inner region is described by Eqn. (7) and Eqn. (8), where the key factors are the pore pressure and Darcy flux. Due to the ill distribution of inner fluid particles, it is unstable to directly compute the pore pressure and Darcy flux on the fluid particles. To overcome this difficulty, we calculate them on the solid particles and then interpolate the results back on the fluid particles. Specifically, for each fluid particle, we assign its fluid mass to the neighbourhood solid particles by computing the SPH kernel sum of nearby solid particles $s$ on fluid particle $f$ :

$$
N_{f}=\sum_{s} W_{f s}
$$

and then computing phase $k$ 's absorbed volume rate as

$$
\beta_{s k}=\frac{1}{\rho_{k} V_{0}} \sum_{f} \frac{\beta_{f k} m_{f} \rho_{k} W_{f s}}{\rho_{f m} N_{f}}
$$

where $V_{0}$ denotes the rest volume of solid particle, and $\rho_{k}$ the rest density of phase $k$. Detailed derivations are given in Appendix A. After computing $\beta_{s k}$ on the solid particles, the solid pore pressure can be obtained as

$$
p^{\text {pore }}=p_{0} \sum_{k} \beta_{s k}-B \epsilon_{s}
$$

where $p_{0}$ is the rest pore pressure, $\epsilon_{s}$ is the volumetric strain and $B$ is a constant value. Representing the interaction between inner flow and porous solid, the above pore pressure $p^{\text {pore }}$ can be substituted into Eqn. (5) to obtain the volumetric strain tensor, and we have $\epsilon_{s}=\operatorname{tr}(\epsilon)$. Captured by Eqn. (11), a porous solid can absorb more fluid when it is stretched; and when the porous solid is squeezed, the volumetric strain will increase the pore pressure to push the inner fluid out.

After computing Darcy flux using Eqn. (1) on the solid particles, the pore velocity, i.e. the velocity of inner fluid relative to the porous solid, can be obtained as $\boldsymbol{u}_{r k}=\frac{1}{e} \boldsymbol{q}_{k}$, where $e$ denotes the porosity of porous solid. Thus, on a solid particle, the velocity of phase $k$ can be updated as:

$$
\boldsymbol{u}_{\beta k}=\boldsymbol{u}_{r k}+\boldsymbol{u}_{s}
$$

which can then be interpolated back to the fluid particles as needed.

The above constitutive models apply to both inner and hybrid fluid particles. For the momentum equation Eqn. (8), since the 
hybrid particles are also affected by the outer-region forces, we universally interpret the inner and outer factors to force sources applied on the whole mixture particle. A pore pressure term $\boldsymbol{a}^{\text {pore }}$ is calculated so that it recovers exactly the influence from each phase when their inner-phase velocity follows Eqn. (12). For simplicity, we directly summarize the result below with the detailed derivation explained in Appendix B:

$$
\boldsymbol{a}^{\text {pore }}=\sum_{k} \frac{\beta_{k}}{\rho_{m} \Delta t}\left(\rho_{k} \boldsymbol{u}_{\beta k}-\rho_{\beta m} \boldsymbol{u}_{m}\right)
$$

where $\rho_{\beta m}=\sum_{k} \frac{\beta_{k} \rho_{k}}{\sum_{k^{\prime}} \beta_{k^{\prime}}}$ is the inner rest density.

\subsection{Outer Region Physics}

Using the virtual phase concept described in $\S 3.2$, we extend the mixture model [Ren et al. 2014; Yan et al. 2016] to handle the multiple-fluid flow in the outer region of porous material. Specifically, the computation of drift velocity is modified as:

$$
\begin{aligned}
\boldsymbol{u}_{m k}= & \tau\left(\rho_{k}-\sum_{k^{\prime}} c_{\alpha k^{\prime}} \rho_{k^{\prime}}\right) \boldsymbol{a}-\tau\left(\nabla p_{k}-\sum_{k^{\prime}} c_{\alpha k^{\prime}} \nabla p_{k^{\prime}}\right)- \\
& \sigma\left(\frac{\nabla \alpha_{k}}{\alpha_{k}}-\sum_{k^{\prime}} c_{\alpha k^{\prime}} \frac{\nabla \alpha_{k^{\prime}}}{\alpha_{k^{\prime}}}\right)-\varphi\left(\nabla p_{k}^{\text {pore }}-\sum_{k^{\prime}} c_{\alpha k^{\prime}} \nabla p_{k^{\prime}}^{\text {pore }}\right)
\end{aligned}
$$

where $c_{\alpha k}=\frac{\alpha_{k} \rho_{k}}{\sum_{k^{\prime}} \alpha_{k^{\prime}} \rho_{\alpha m}}$ denotes the outer mass fraction, $\rho_{\alpha m}=$ $\sum_{k} \frac{\alpha_{k} \rho_{k}}{\sum_{k^{\prime}} \alpha_{k^{\prime}}}$ denotes the outer mixture density, $\boldsymbol{a}=g-\left(\boldsymbol{u}_{m} \cdot \nabla\right) \boldsymbol{u}_{m}-$ $\frac{\partial \boldsymbol{u}_{m}}{\partial t}$, and $\tau, \sigma$ and $\varphi$ are constant weight factors. The first three terms denote the velocity difference caused by the body force, pressure and diffusion effects, respectively, and the last term represents the influence of liquid absorption and emission by the porous solid.

\subsection{Absorption and Capillary Effects}

Liquid absorption and emission can occur on the surface of porous solid. To capture these effects, we assume that the outer fluid on the solid surface also has a constant pore pressure. When the outer pore pressure is greater than the inner pore pressure, the liquid will get absorbed and a liquid particle may flow into the solid, and vice versa. Inside the porous solid, the pore pressure is unified and related to the absorbed fluid volume and volumetric strain by Eqn. (11). Note that different fluid phases in the outer region may have different pore pressures. The pore pressure for phase $k$ in the outer region is given by:

$$
p_{k}^{\text {pore }}=p_{0} e \theta_{k}
$$

where $\theta_{k}$ is an absorption constant.

Similar to the Darcy flux calculation for the inner region Eqn. (1), we compute the gradient of pore pressure to obtain Darcy flux on the solid surface:

$$
D_{k}=-K \frac{\boldsymbol{k}_{\boldsymbol{w}}}{\mu_{k}}\left(\alpha_{k}+\beta_{k}\right) \nabla p_{k}^{\text {pore }}
$$

where $K$ is a constant capillary ratio coefficient related to the solid. The dimensionless term $K\left(\alpha_{k}+\beta_{k}\right)$ is multiplied to avoid creating fake flux. Then, Eqn. (7) and Eqn. (6) are used to determine the exchange of internal and external fluids.

When a part of fluid mass is absorbed by the solid, the fluid particle will be subject to capillary force near the solid surface, preventing liquid escaping from the porous solid. We use the same model as [Becker and Teschner 2007] to capture the capillary force:

$$
\boldsymbol{a}^{c a p}=\psi \sum_{s} \sum_{k} \beta_{f k} m_{s} \nabla W_{f s}
$$

where $\psi$ is a constant strength factor. Near the porous surface, the capillary force generates an inward dragging effect. After the particle flows into the solid, it will gradually reduce to zero so that the motion of fluid particles gets purely governed by Darcy's law.

\section{IMPLEMENTATION}

The implementation details of the proposed unified particle model for multiple-fluid flow and porous material are explained in this section. §5.1 explains the numerics for pressure gradient and Darcy flux, and $\S 5.2$ the algorithm framework.

\subsection{Pressure Gradient and Darcy Flux}

For each solid or fluid particle $i$, the interpolated density is computed by $\rho_{i}=\sum_{j} m_{j} W_{i j}$, where $j$ denotes the nearby particle. In addition, since the inner fluid particles flow inside the solid, we compute the solid density using $\rho_{s}^{\prime}=\sum_{s_{s}} m_{0} W_{s s_{s}}$ in order to achieve a stable interpolation. For each solid particle $s$, the gradient of pore pressure is computed by:

$$
\nabla p_{s}^{\text {pore }}=\sum_{s_{s}} \frac{m_{s_{s}}}{\rho_{s_{s}}^{\prime}}\left(p_{s}^{\text {pore }}-p_{s_{s}}^{\text {pore }}\right) \nabla W_{s s_{s}}
$$

where $s_{s}$ denotes the nearby solid particles. Then the collection of inner phase $k$ 's velocity from solid particles to fluid particles is given by

$$
\boldsymbol{u}_{\beta k}=\sum_{s}\left(\boldsymbol{u}_{r k}+\boldsymbol{u}_{s}\right) W_{f s} / N_{f}
$$

where $N_{f}$ is the same with that in Eqn. (9). The $\boldsymbol{u}_{\beta k}$ is then used for Eqn. (13). A special case is $N_{f}=0$, for which we simply set $a^{\text {pore }}=0$.

For each fluid particle $f$, only the nearby solid particles are considered:

$$
D_{k}=-K \frac{\boldsymbol{k}_{\boldsymbol{w} \boldsymbol{k}}}{\mu_{k}} \sum_{s} \frac{m_{s}}{\rho_{s}}\left(\alpha_{f k}+\beta_{s k}\right)\left(p_{f k}^{\text {pore }}-p_{s}^{\text {pore }}\right) \nabla W_{f s}
$$

Then we compute the source term using

$$
\nabla \cdot \boldsymbol{D}_{k}=\sum_{s} \frac{m_{s}}{\rho_{s}} \boldsymbol{D}_{k} \cdot \nabla W_{f s}
$$

For Eqn. (14), the gradient of outer fluid pore pressure is computed by

$$
\nabla p_{k}^{\text {pore }}=\sum_{s} \frac{m_{s}}{\rho_{s}}\left(p_{f k}^{\text {pore }}-p_{s}^{\text {pore }}\right) \nabla W_{f s}
$$

\subsection{Algorithm Framework}

The algorithm can be divided into three stages: the outer-region pressure stage, the inner-region momentum stage and the integration stage, which are performed on all fluid or solid particles without labelling particle regions. The overall algorithm framework is shown in Algorithm 1. 

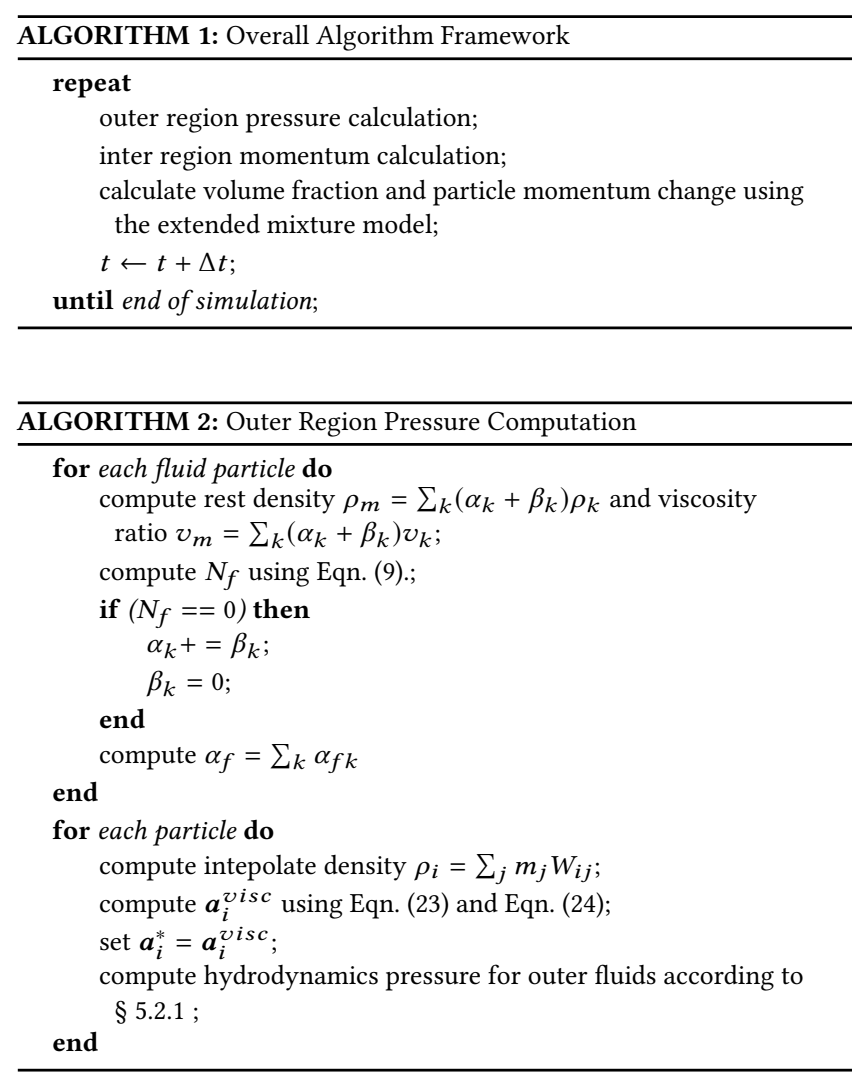

5.2.1 Outer Region Pressure Computation. Instead of using the state equation based approach as in [Ren et al. 2014; Yan et al. 2016], we use an IISPH-like scheme to compute the hydrodynamic pressure for the outer-region fluid particles, which gives better incompressibility. The algorithm workflow is given in Algorithm 2. We adopt the same algorithm framework as [Band et al. 2018] to compute pressure and implement two-way coupling. A series of modifications are made to support multiple-fluid porous flow simulation.

First, based on [Yan et al. 2016], the fluid viscosity is obtained as

$$
\boldsymbol{a}_{i}^{v i s c}=\sum_{f} \sum_{k} \frac{\alpha_{i k} \alpha_{f k}}{\alpha_{i k}+\alpha_{f k}}\left(\boldsymbol{u}_{i}-\boldsymbol{u}_{f}\right) \frac{m_{f}}{\rho_{i} \rho_{f}}\left(v_{m i}+v_{m f}\right) W_{i f}
$$

The viscosity of the elastic solid particle is:

$$
\boldsymbol{a}_{i}^{v i s c}=\sum_{s} \sum_{k}\left(\boldsymbol{u}_{i}-\boldsymbol{u}_{s}\right) \frac{m_{s}}{\rho_{i} \rho_{s}}\left(v_{m i}+v_{m s}\right) W_{i s}
$$

Then the intermediate acceleration of liquid and solid particles is set as $\boldsymbol{a}_{i}^{*}=\boldsymbol{a}_{i}^{v i s c}$. Note that for the outer region calculation, solid particles act as scene boundary for liquid pressure calculation. Secondly, the rest volume of the liquid particles are updated to the effective volume of outer liquid to exclude the absorbed fluid in the computation. At the beginning of each time step, we calculate $\alpha_{f}=\sum_{k} \alpha_{k}$ for each fluid particle, after which its rest volume is approximated by $V_{f 0}=\alpha_{f} h^{3}$. Since this volume of fluid particles near the solid varies greatly, we use the harmonic mean for better stability, so that the divergence of velocity becomes

$$
\begin{aligned}
& \nabla \cdot \boldsymbol{u}_{f}^{*}=-\sum_{j} \frac{V_{f} V_{j}}{V_{f}+V_{j}}\left(\boldsymbol{u}_{f}^{*}-\boldsymbol{u}_{j}^{*}\right) \nabla W_{f j} \\
& \nabla \cdot \boldsymbol{u}_{s}^{*}=-\sum_{f} \frac{V_{f} V_{s}}{V_{f}+V_{s}}\left(\boldsymbol{u}_{s}^{*}-\boldsymbol{u}_{f}^{*}\right) \nabla W_{s f}
\end{aligned}
$$

The same harmonic interpolation also applies to the following terms as in [Band et al. 2018]:

$$
\begin{gathered}
\boldsymbol{a}_{i}^{\text {press }}=-\frac{V_{i}}{m_{i}} \sum_{j} \frac{V_{i} V_{j}}{V_{i}+V_{j}}\left(p_{i}+p_{j}\right) \nabla W_{i j} \\
(A p)_{i}=\Delta t^{2} \sum_{j} \frac{V_{i} V_{j}}{V_{i}+V_{j}}\left(\boldsymbol{a}_{i}^{\text {press }}-\boldsymbol{a}_{j}^{\text {press }}\right) \cdot \nabla W_{i j}
\end{gathered}
$$

In our simulator we do not explicitly track the position state of fluid particles. To ensure the above hydrodynamics pressure only applies to the outer region fluids, we further multiply the term $S_{f}$ in [Band et al. 2018] by $\alpha_{i}$ so that the inner fluid particles will only be affected by pore pressure and gravity:

$$
S_{f}=\alpha_{f}\left(1-\frac{V_{f 0}}{V_{f}}+\Delta \cdot \boldsymbol{u}_{f}^{*}\right)
$$

5.2.2 Inner Region Momentum Computation. Darcy's law is used to simulate multiple fluid porous flow in the inner region, and the workflow is given in Algorithm 3.

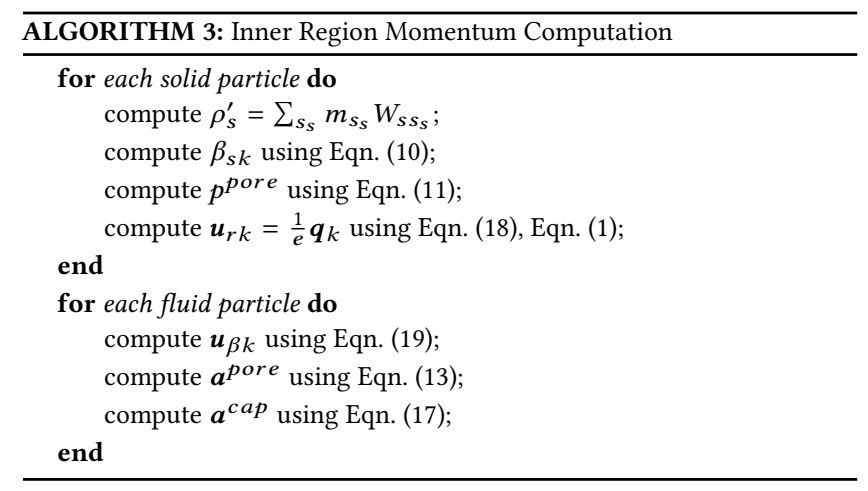

5.2.3 Time Integration. After computing the absorption term and drift velocity, we integrate the volume fraction and momentum governing equations as detailed in Algorithm 4.

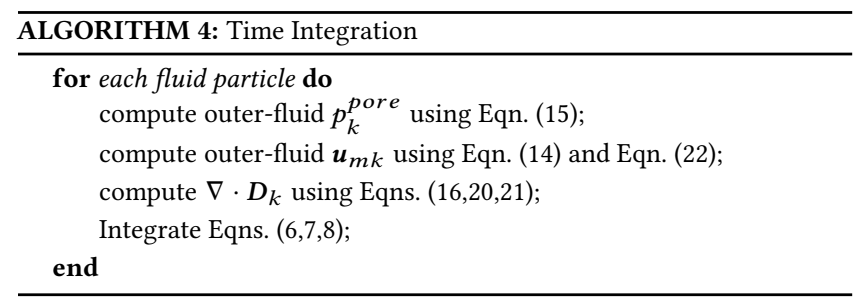


Table 1: Performance Data

\begin{tabular}{l|c|c|c|c}
\hline & $\begin{array}{c}\text { Liquid } \\
\text { Phases }\end{array}$ & $\begin{array}{c}\text { Liquid } \\
\text { Particles }\end{array}$ & $\begin{array}{c}\text { Solid } \\
\text { Particles }\end{array}$ & $\begin{array}{c}\text { runtime } \\
\text { (second/step) }\end{array}$ \\
\hline Example 1 & 1 & 272,000 & 247,000 & 0.21 \\
Example 2 & 2 & 879,000 & 94,600 & 0.34 \\
Example 3 & 1 & 864,000 & 41,000 & 0.65 \\
Example 4 & 2 & $1,091,000$ & 504,000 & 0.44 \\
Example 5 & 3 & 299,000 & 346,000 & 0.27 \\
\hline
\end{tabular}

\section{RESULTS}

We implement the proposed unified particle model for multiple-fluid flow and porous material on a Nvidia GeForce GTX 1080Ti GPU. In all examples, we generally set $e=0.6, K=0.01, \boldsymbol{k}_{\boldsymbol{w} \boldsymbol{k}}=10^{-11} \boldsymbol{I}$, $B=0.003, \varphi=2 \times 10^{-12}$, and $p_{0}=4.8 \times 10^{9}$. The performance data of all examples are recorded in Table 1.

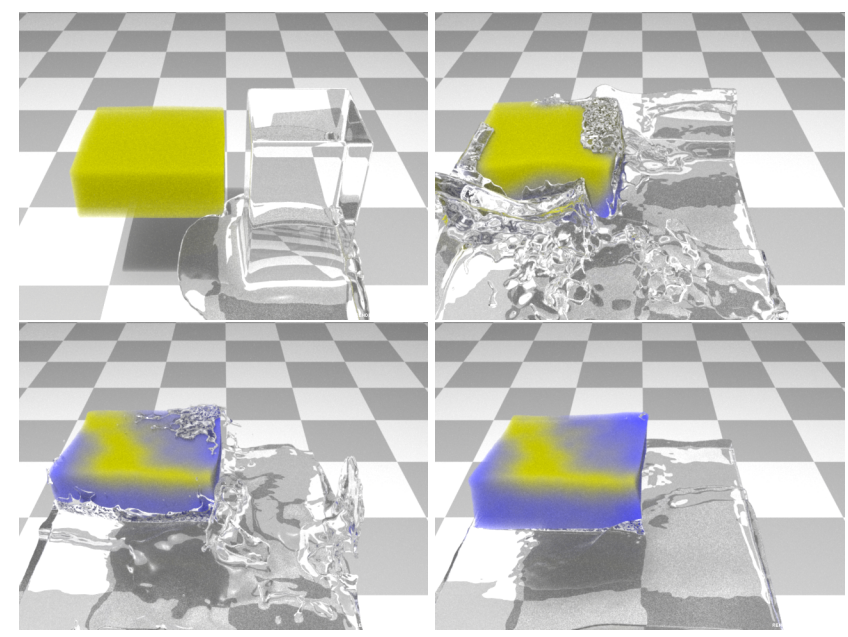

Figure 2: Single fluid porous flow. The regions where liquid is absorbed are rendered in blue colour.

Example 1 (Fig. 2) shows a porous block absorbing a single-fluid liquid. When the flow wave reaches the solid surface, it causes distortion of the solid body and the flow is bounced back. At the same time some of the liquid gets absorbed into the porous material, leaving visual traces when the bulk liquid drops back. The wet regions of the solid also expand in volume showing poroelasticity effect. Since no particle deletion or insertion is needed, our approach automatically guarantees the mass conservation throughout the simulation.

Example 2 (Fig. 3) shows a porous solid in a two-phase flow. The two fluid phases are mixed as $1: 1$ volume fraction, but only the red liquid can be absorbed by the porous solid. Using the proposed virtual phase concept, complex coupling of different governing equations in inner and outer regions is resolved. Consistent mass and momentum transport is achieved without particle splitting, with only red phase entering the solid and the green phase being left outside. During the simulation the duck is squeezed, pushing some red liquid out from the solid. The capillary effect at porous surface prevents the red liquid from free escape.

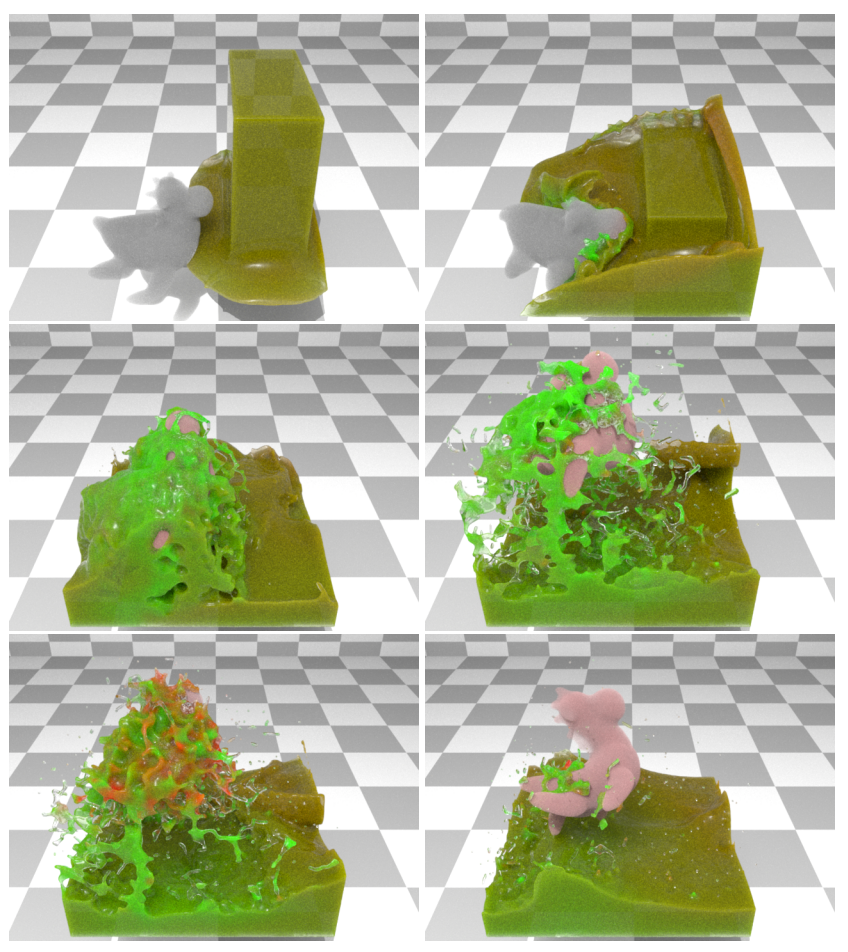

Figure 3: A two-phase mixture (red and green) interacts with a porous duck. Initially, the mixture turns into green around the porous duck (first row), because only the red phase can be absorbed into the porous solid. Then, the duck is lifted out of the liquid mixture (second row) and squeezed (third row), causing some red phase to come out and remix into the liquid.

Example 3 (Fig. 4) demonstrates the solid-particle properties can be influenced by absorbing the liquid. Initially, the porous balance stick has uniform density and is lighter than the liquid. Then, the purple side becomes heavier after absorbing liquid and drags the whole stick into the tank. The stick is slightly stretched during this process. To achieve this effect, we only need to adjust the mass for solid particles so that they increase with the $\beta_{s k}$ value, i.e. adding the absorbed liquid mass.

In Example 4 (Fig. 5), two fluid phases pass through a perforated material separately. The two fluid phases are absorbed differently by the solid, and as a result the red phase is largely trapped in the solid while the less-absorbed green phase falls almost freely through the perforated material.

In Example 5 (Fig. 1) a three-phase mixture is poured onto a stack of three porous solids, where each porous solid can only absorb one specific liquid phase. The liquid mixture first builds up on the top of solid stack due to the resistance from the porous solids. Then, as each liquid phase passes through and gets absorbed by the porous solid, both the liquid mixture and the porous solids change colour. Finally, the liquid at the bottom of container shows a blueish colour, which corresponds to the less absorbed phases. 


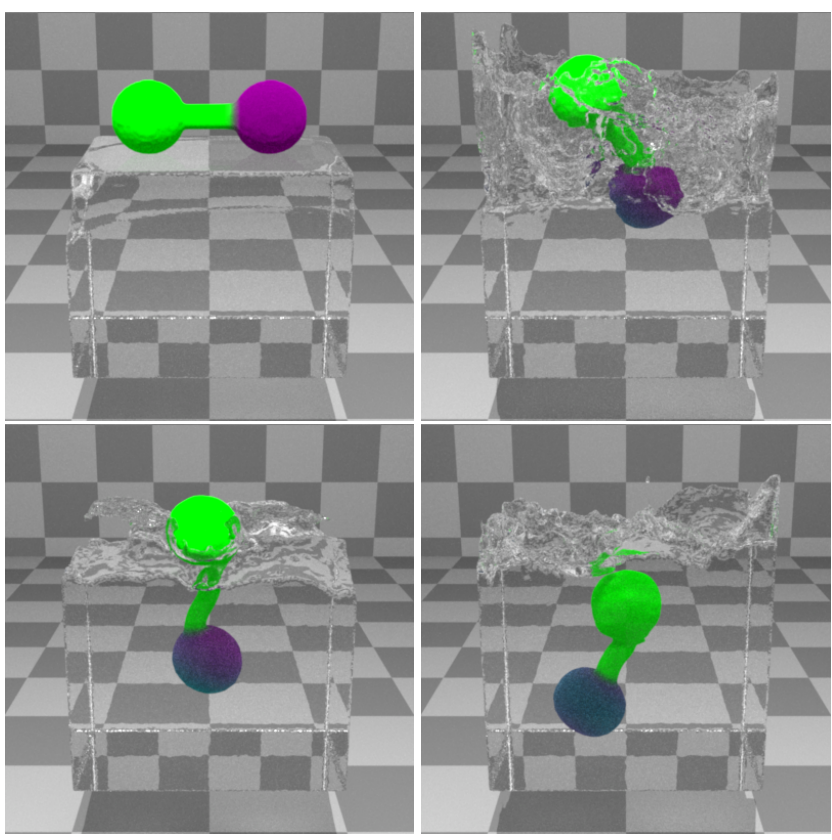

Figure 4: Solid property affected by liquid absorption. The purple end of the stick becomes heavier after absorbing the liquid, dragging the solid into the tank.
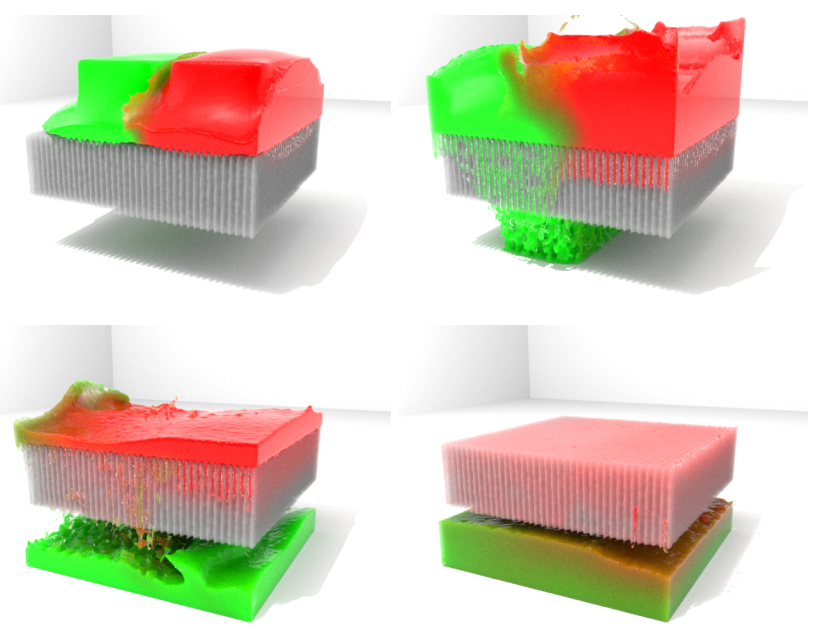

Figure 5: Two fluid phases passes through a perforated material. The less-absorbed green phase passes the solid region faster, leaving the red phase behind.

\section{CONCLUSION}

We present an SPH-based simulation scheme for multiple-fluid liquid flow interacting with sponge-like porous materials. The SPH fluid particles are universally handled without exhausting deleting or splitting process at the solid surface. Our approach applies to multiple fluid simulations and ensures physically consistent mass and momentum transport.
The simplification made in the proposed approach also causes some limitations. Darcy's law does not support free flow inside the porous material, and is unsuitable for materials with larger voids or fractures. In addition, assigning a constant outer-region pore pressure for each phase during solid-surface absorption (Eqn.(15)) is a simple and effective treatment, but it ignores other more complex absorption effects that could occur when the multiple fluids and solids behave differently. A possible solution involves decoupling the pore pressure in the solid, which we would like to investigate in the future. The adjustment of solid particle mass slows the convergence of the implicit pressure and solid solvers and increases the simulation time, as is shown in Table 1. Further investigations into derivations of the implicit solvers may enhance the performance in such cases.

\section{REFERENCES}

Stefan Band, Christoph Gissler, Markus Ihmsen, Jens Cornelis, Andreas Peer, and Matthias Teschner. 2018. Pressure Boundaries for Implicit Incompressible SPH. ACM Trans. Graph. 37, 2, Article 14 (Feb. 2018), 11 pages. https://doi.org/10.1145/3180486

Markus Becker and Matthias Teschner. 2007. Weakly compressible SPH for free surface flows. In Proceedings of the 2007 ACM SIGGRAPH/Eurographics symposium on Computer animation. Eurographics Association, 209-217.

Stefano Berrone, Sandra Pieraccini, and Stefano Scialò. 2017. Flow simulations in porous media with immersed intersecting fractures. F. Comput. Phys. 345 (2017), 768 - 791. https://doi.org/10.1016/j.jcp.2017.05.049

Henry Philibert Gaspard Darcy. 1856. Les Fontaines publiques de la ville de Dijon. Exposition et application des principes à suivre et des formules à employer dans les questions de distribution d'eau, etc. V. Dalamont.

Ming Gao, Andre Pradhana, Xuchen Han, Qi Guo, Grant Kot, Eftychios Sifakis, and Chenfanfu Jiang. 2018. Animating Fluid Sediment Mixture in Particle-Laden Flows. ACM Trans. Graph. 37, 4, Article 149 (July 2018), 11 pages. https://doi.org/10.1145/ 3197517.3201309

R Hilfer. 2006. Macroscopic capillarity and hysteresis for flow in porous media. Physical review. E, Statistical, nonlinear, and soft matter physics 73 (02 2006), 016307. https: //doi.org/10.1103/PhysRevE.73.016307

Toon Lenaerts, Bart Adams, and Philip Dutré. 2008. Porous Flow in Particle-Based Fluid Simulations. ACM Trans. Graph. 27, 3 (Aug. 2008), 1-8. https://doi.org/10. 1145/1360612.1360648

Andreas Peer, Christoph Gissler, Stefan Band, and Matthias Teschner. 2018. An Implicit SPH Formulation for Incompressible Linearly Elastic Solids. Computer Graphics Forum 37, 6 (2018), 135-148. https://doi.org/10.1111/cgf.13317 arXiv:https://onlinelibrary.wiley.com/doi/pdf/10.1111/cgf.13317

Bo Ren, Chenfeng Li, Xiao Yan, Ming C. Lin, Javier Bonet, and Shi-Min Hu. 2014. Multiple-Fluid SPH Simulation Using a Mixture Model. ACM Trans. Graph. 33, 5, Article 171 (Sept. 2014), 11 pages. https://doi.org/10.1145/2645703

Andre Pradhana Tampubolon, Theodore Gast, Gergely Klár, Chuyuan Fu, Joseph Teran, Chenfanfu Jiang, and Ken Museth. 2017. Multi-Species Simulation of Porous Sand and Water Mixtures. ACM Trans. Graph. 36, 4, Article 105 (July 2017), 11 pages. https://doi.org/10.1145/3072959.3073651

Yu-Shu Wu, Lehua Pan, and Karsten Pruess. 2004. A physically based approach for modeling multiphase fracture-matrix interaction in fractured porous media. Advances in Water Resources 27, 9 (2004), 875 - 887. https://doi.org/10.1016/j. advwatres.2004.07.002

Xiao Yan, Yun-Tao Jiang, Chen-Feng Li, Ralph R. Martin, and Shi-Min Hu. 2016. Multiphase SPH Simulation for Interactive Fluids and Solids. ACM Trans. Graph. 35, 4, Article 79 (July 2016), 11 pages. https://doi.org/10.1145/2897824.2925897

Tao Yang, Jian Chang, Ming C. Lin, Ralph R. Martin, Jian J. Zhang, and Shi-Min Hu. 2017. A Unified Particle System Framework for Multi-Phase, Multi-Material Visual Simulations. ACM Trans. Graph. 36, 6, Article 224 (Nov. 2017), 13 pages. https://doi.org/10.1145/3130800.3130882

\section{A ABSORBED VOLUME RATE}

To compute the absorbed volume rate, we set $m_{s k}$ as the phase $k$ 's absorbed mass on solid particle $s$ and $m_{f k}$ as the phase $k$ 's absorbed mass on fluid particle. First, the following relations hold for $m_{f k}$ to 
$m_{s k}:$

$$
\begin{gathered}
m_{s k}=\sum_{f} \frac{m_{f k} W_{f s}}{N_{f}} \\
m_{f k}=\frac{\beta_{f k} \rho_{f k}}{\rho_{f m}} m_{f}
\end{gathered}
$$

which can be merged and simplified to

$$
m_{s k}=\sum_{f} \frac{\beta_{f k} \rho_{f k} m_{f} W_{f s}}{\rho_{f m} N_{f}}
$$

Then we can obtain

$$
\beta_{s k}=\frac{m_{s k}}{\rho_{k} V_{0}}
$$

where $V_{0}$ is the rest volume of the solid particle given in the initialization.

\section{B PORE ACCELERATION}

For each fluid particle, each phase has individual phase velocity and fluid particle moves according to the mixture velocity $\boldsymbol{u}_{m}$. The mixture velocity is computed by $\boldsymbol{u}_{m}=\frac{1}{\rho_{m}} \sum_{k} \rho_{k}\left(\alpha_{k} \boldsymbol{u}_{\alpha k}+\beta_{k} \boldsymbol{u}_{\beta k}\right)$, and the mixture density is computed by $\rho_{m}=\sum_{k}\left(\alpha_{k}+\beta_{k}\right) \rho_{k}$.

We first equate the impulse of desired inner forces to the momentum change of inner mixture:

$$
\sum_{k} \rho_{k} \beta_{k} V \boldsymbol{a}_{k}^{*} \Delta t=\sum_{k} \rho_{k} \beta_{k} V \boldsymbol{u}_{\beta k}-\rho_{\beta m} \sum_{k} \beta_{k} V \boldsymbol{u}_{m}
$$

where $\rho_{\beta m}=\sum_{k} \frac{\beta_{k} \rho_{k}}{\sum_{k^{\prime}} \beta_{k^{\prime}}}$. A solution to this equation is given by:

$$
\boldsymbol{a}_{k}^{*}=\frac{\rho_{k} \boldsymbol{u}_{\beta k}-\rho_{\beta m} \boldsymbol{u}_{m}}{\rho_{k} \Delta t}
$$

Then, assuming

$$
\rho_{m} V \boldsymbol{a}^{\text {pore }}=\sum_{k} \rho_{k} \beta_{k} V \boldsymbol{a}_{k}^{*}
$$

we can obtain the $\boldsymbol{a}^{\text {pore }}$ term in Eqn. (13). 